\title{
Ultrasound-guided serratus plane block as an effective adjunct to systemic analgesia in four dogs undergoing thoracotomy
}

\author{
Iago Asorey*, Beatrice Sambugaro, Rebecca J. Bhalla and Maja Drozdzynska \\ Dick White Referrals, Station Farm, London Road, Six Mile Bottom, Cambridgeshire, CB8 0UH, UK
}

\begin{abstract}
Background: Ultrasound-guided serratus plane block (UGSPB) is a loco-regional anesthesia technique designed to desensitize the thoracic wall. It is a compartmental block, where local anesthetic is delivered to the fascial, intermuscular plane. Since its original description in humans, two cadaveric veterinary studies, redefining the technique, have been performed. Taking into account the successful use of the UGSPB in human medicine, we employed the veterinary description to perform this block in four dogs undergoing thoracotomy. The case series described below aims to share our experience of the clinical application of this new loco-regional anesthesia technique in dogs.

Case Description: Four dogs, with different underlying medical conditions underwent cranial lateral thoracotomy. The analgesia protocol consisted of intravenous methadone and UGSPB performed half an hour before the beginning of the procedure. The cardiovascular system was closely monitored for any signs of nociception. Fentanyl, although available as rescue analgesia, was not required in any of these cases as no signs of nociception were present.

Conclusion: To the authors' knowledge, this is the first veterinary clinical report using the UGSPB as a part of a multimodal analgesia protocol in dogs undergoing thoracotomy. Based on this observation, UGSPB has the potential to prevent nociception and reduce the intraoperative opioid requirements in dogs undergoing thoracotomy. A prospective randomized clinical trial is required to confirm these promising results.
\end{abstract}

Keywords: Dog, Levobupivacaine, Multimodal analgesia, Thoracotomy, Ultrasound-guided serratus plane block.

\section{Introduction}

In dogs, lateral thoracotomy has been widely used for surgical management of different thoracic conditions (Moores et al., 2007). Patients undergoing lateral thoracotomy can experience severe acute pain caused by rib retraction, damage to serratus/intercostal muscles and intercostal nerves. The unique feature of thoracotomies is that unsuccessful treatment of pain will lead to immediate postoperative complications like hypoventilation and possible hypoxemia (Berg and Orton 1986; Conzemius et al., 1994; Stobie et al., 1995). Unlike abdominal procedures, chest surgeries require immediate recovery of the dynamic function of the respiratory muscles. That presents the additional challenge for the anesthetist of providing not only static but also dynamic analgesia. Moreover, thoracotomies are more commonly associated with chronic pain conditions in humans. Post-thoracotomy pain syndrome affects $25 \%-60 \%$ of people undergoing thoracotomy (Wildgaard et al., 2009). Although its incidence is currently unknown in veterinary clinical practice, experimental studies in rats confirmed its existence (Chi-Fei Wang et al., 2013). With the aim to reduce or even prevent these acute and chronic complications, loco-regional analgesic techniques can be performed as a complement or alternative to systemic analgesia. The ultrasound-guided serratus plane block (UGSPB) is described in humans as an alternative loco-regional technique for thoracic wall procedures (Blanco et al., 2013). Two approaches for this block have been described in humans and dogs: superficial (Blanco et al., 2013; Freitag et al., 2019) and deep (Blanco et al., 2013; Drozdzynska et al., 2017). Briefly, this compartmental block is based on the injection of local anesthetic solution superficial or deep to the serratus muscle aiming to block the lateral cutaneous branches of the intercostal nerves. Although, the superficial technique seemed to result in more effective sensory block while tested in human volunteers that did not undergo surgery (Blanco et al., 2013), another study performed in women undergoing breast cancer surgery concluded that deep UGSPB is as effective as the superficial technique (Abdallah et al., 2018). In human medicine, to date, UGSPB has been used as an effective analgesic technique for the treatment of pain associated with multiple rib fractures, thoracotomy, mastectomy, and thoracoscopy (Durant et al., 2017; Ökmen and Ökmen 2017; Zocca et al., 2017; Kim et al., 2018).

The deep UGSPB has been described in a canine cadaveric study (Drozdzynska et al., 2017). The authors concluded that $1 \mathrm{ml} / \mathrm{kg}$ of dye-contrast solution administered between the serratus ventralis muscle (SVM) and external intercostal muscles (EIMs) allows for distribution of contrast within one plane over the cranial thoracic wall between the first and sixth intercostal space. In this study, two injection points with equal volumes $(0.5$ $\mathrm{ml} / \mathrm{kg}$ ) were used. First in the 4th intercostal space at the level of mid-thorax just below the ventral border of the scapula and second in the dorsal thorax at the level of 5th intercostal space (Drozdzynska et al., 2017). 
Although the UGSPB is potentially suitable for the prevention and treatment of pain associated with the lateral thoracic wall trauma due to surgery, there are currently no clinical reports of this block in veterinary medicine. The aim of this case series is to describe the results of deep UGSPB use in four dogs that underwent lateral thoracotomy.

\section{Case Details}

The first case was an 8-year-old male Staffordshire Bull Terrier weighing $22.3 \mathrm{~kg}$ diagnosed with a lung tumor. A right-sided thoracotomy was performed at the sixth intercostal space for right caudal lung lobectomy. The dog was premedicated with dexmedetomidine (Dexdomitor ${ }^{\circledR}$; Orion Pharma, UK) $1 \mu \mathrm{g} / \mathrm{kg}$ and methadone (Comfortan ${ }^{\circledR}$; Dechra, UK) $0.2 \mathrm{mg} / \mathrm{kg} \mathrm{IV}$. Anesthesia was induced with propofol (PropoFlo®; Zoetis, UK) $2.7 \mathrm{mg} / \mathrm{kg}$ and lidocaine (Lidocaine Hydrochloride; Hameln, UK) $0.9 \mathrm{mg} / \mathrm{kg}$ IV and maintained by total intravenous anesthesia (TIVA) of propofol (Fresenius Propoven $\AA$; Fresenius Kabi, UK) at $0.1-0.2 \mathrm{mg} / \mathrm{kg} / \mathrm{minute}$. The dog was positioned in left lateral recumbency with the uppermost thoracic limb extended cranially. After clipping and aseptic skin preparation, UGSPB was performed. The linear ultrasound probe (5-13 MHz; SonoScape ${ }^{\mathrm{TM}}$, Shenzhen, China) was positioned perpendicularly to the long axis of the ribs over the mid-dorsal chest at the level of the fourth and later fifth intercostal space. The plane between the EIM and SVM was identified (Fig. 1). Using a 22 gauge, $38 \mathrm{~mm}$ hypodermic needle (Terumo®; Hamburg, Germany), levobupivacaine (Chirocaine ${ }^{\circledR}$; AbbVie, UK) $0.25 \%, 1 \mathrm{ml} / \mathrm{kg}$ was injected using an inplane technique. The first injection was performed at the level of the fourth intercostal space and the second at the level of fifth intercostal space $(0.5 \mathrm{ml} / \mathrm{kg}$ at each site), as described by Drozdzynska et al. (2017). Each time, hydro-dissection of the fascial plane (FP) was seen and interpreted as correct placement of the needle tip.

Case 2 was a 10-year-old male Labrador Retriever with a body weight of $38.9 \mathrm{~kg}$ who was diagnosed with a left cranial lung lobe nodule and a heart base tumor. The dog underwent a right fourth intercostal space thoracotomy for left cranial lung lobectomy and subtotal pericardiectomy. Dexmedetomidine $1 \mu \mathrm{g} / \mathrm{kg}$ and methadone $0.2 \mathrm{mg} / \mathrm{kg}$ were administered IV as premedication. Anesthesia was induced with propofol $3 \mathrm{mg} / \mathrm{kg}$ IV and maintained with propofol TIVA (0.3-0.4 $\mathrm{mg} / \mathrm{kg} / \mathrm{minute})$. The UGSPB was performed as previously described with $1 \mathrm{ml} / \mathrm{kg}$ of $0.25 \%$ levobupivacaine.

The third case was a 9-month-old male Cavalier King Charles Spaniel weighing $8.0 \mathrm{~kg}$ who, following traumatic chest injury, underwent a right fifth intercostal space exploratory thoracotomy for rib repair surgery. The dog was premedicated with methadone $0.1 \mathrm{mg} / \mathrm{kg}$ IV. Anesthesia was induced with propofol $3.8 \mathrm{mg} / \mathrm{kg}$

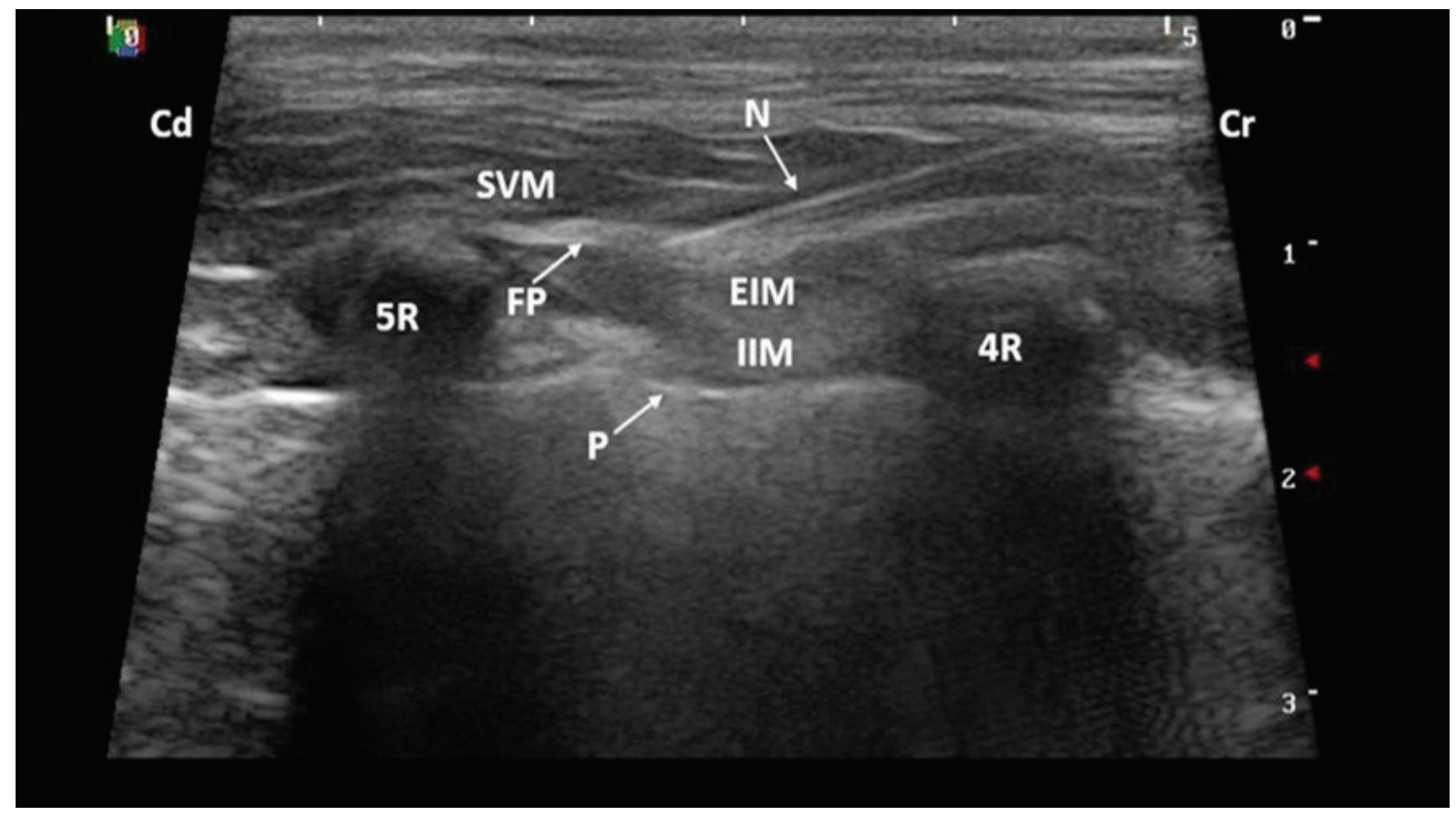

Fig. 1. Ultrasound image taken with the dog in lateral recumbency with a 5-13 MHz linear probe positioned perpendicular to the long axis of the ribs over the mid-dorsal thorax at the level of the fourth intercostal space. A 22 gauge, 38 mm hypodermic needle was inserted in-plane and advanced cranio-caudally aiming to locate the tip in the FP between the SVM and EIM. (4R): fourth rib; (5R): fifth rib; (Cd): caudal; (Cr): cranial; (EIM): external intercostal muscles; (FP): fascial plane; (IIM): internal intercostal muscle; $(\mathrm{N})$ : needle; $(\mathrm{P})$ : pleura; $(\mathrm{SVM})$ : serratus ventralis muscle. 
IV and maintained with Isoflurane (IsoFlo®; Zoetis, UK) vaporized in oxygen (end-tidal Isoflurane 1.2\%1.3\%; Wato EX-65; Mindray, China). The UGSPB was performed with $1.2 \mathrm{ml} / \mathrm{kg}$ of $0.125 \%$ levobupivacaine.

Case 4 involved an 11-year-old female Yorkshire Terrier with a body weight of $4.1 \mathrm{~kg}$ who underwent a left-sided fifth intercostal space thoracotomy for subtotal pericardiectomy due to the recurrent pericardial effusion. The dog was premedicated with methadone $0.1 \mathrm{mg} / \mathrm{kg} \mathrm{IV}$, and anesthesia was induced with midazolam (Hypnovel@; Roche, UK) $0.2 \mathrm{mg} / \mathrm{kg}$ and alfaxalone (Alfaxan ${ }^{\circledR}$; Jurox, UK) $1 \mathrm{mg} / \mathrm{kg}$ both given IV and maintained with Sevoflurane (SevoFlo ${ }^{\circledR}$; Zoetis, UK) vaporized in oxygen (end-tidal Sevoflurane $2.2-2.3 \%$ ). The UGSPB was performed with $1.9 \mathrm{ml} / \mathrm{kg}$ of $0.15 \%$ levobupivacaine.

Intraoperative nociception is defined as $20 \%$ increase in the heart rate or blood pressure values in comparison to baseline (Mansour et al., 2017). Such a nociceptive response would require rescue analgesia. Patients reported here had changes in heart rate and blood pressure of less than $10 \%$ throughout the surgical procedure. Thus, none of them received rescue analgesia. In addition, hemodynamic stability was maintained during surgery and intraoperative hypotension (mean arterial blood pressure $\leq 65$ $\mathrm{mmHg}$ ), measured invasively, was not detected in any of the presented cases (Ruffato et al., 2015). Besides, no complications were identified from UGSPB performance. All dogs had smooth anesthetic recovery. Postoperatively, dogs were pain scored using the short form Glasgow Composite pain scale every 2 hours for 24 hours. Postoperative analgesia aimed to maintain the pain score below $6 / 24$. This was managed differently among the patients due to the different recommendations given by the different anesthetists responsible for the cases. Cases 3 and 4 received methadone $(0.1-0.2 \mathrm{mg} / \mathrm{kg})$ IV every 4 hours and interpleural levobupivacaine $1 \mathrm{mg} / \mathrm{kg}$ every 6 hours, both drugs regardless based on the pain scores. Cases 1 and 2 received methadone only when the pain scores were $\geq 6 / 24$. Non-steroidal anti-inflammatory agents were given to all patients. Robenacoxib (Onsior ${ }^{\circledR}$, Elanco, Germany; $1 \mathrm{mg} / \mathrm{kg}$ ) PO q24h in the first case and meloxicam (Metacam ${ }^{\circledR}$, Boehringer Ingelheim, Germany; $0.1 \mathrm{mg} / \mathrm{kg}$ ) PO q24h in the remaining cases.

\section{Discussion}

In the author's experience, lateral thoracotomy causes nociception that is commonly controlled by the constant rate infusion of opioids. It is very unlikely this level of nociception could be controlled with only the low doses of methadone given as a premedication in these cases. This report suggests that UGSPB is an effective adjunct to systemic analgesia for thoracotomy, which controls surgical nociception and allows for significant reduction of intraoperative opioid requirements. The same clinical observation was made in a case series of children undergoing thoracotomy where UGSPB provided very good perioperative analgesia (Biswas et al., 2018a).

In dogs, the serratus ventralis thoracis muscle has its cranial attachment on the serrated part of the scapula and caudal attachment on the seventh to eighth rib, covering the cranial half of the lateral thoracic wall. Ventrally, it extends to the scalenus and external oblique abdominal muscles whereas medially it lies on the EIMs (Evans and de Lahunta, 2012). At the ventral edge of the SVM, the lateral cutaneous branches of intercostal nerves (T4-T7), intercostobrachial nerve III, and long thoracic nerve can be localized (Freitag et al., 2019). The sensory innervation of the thoracic wall is provided by the intercostal nerves which are the ventral branches of the thoracic spinal nerves. Each intercostal nerve runs below the corresponding rib within the neuromuscular bundle and gives rise to a lateral branch that passes through the mid-lateral portion of the thoracic wall. The lateral branch ends by dividing into a middle muscular branch that innervates the thoracic parts of the dorsal abdominal muscles and the lateral cutaneous branch that supplies the skin of the ventrolateral part of the thorax (Evans and de Lahunta, 2012). In dogs, it has been hypothesized that thoracic wall analgesia can be achieved by successful blockade of the lateral cutaneous branches of the intercostal nerves T2 to T11 (Bailey et al., 1984). For this reason, the UGSPB may offer the possibility of blocking the lateral cutaneous branches of the intercostal nerves as suggested in humans, rather than the intercostal nerves themselves (Mayes et al., 2016).

Whenever compartmental blocks are planned, three factors must be considered: the maximum allowed dose of local anesthetic, the total volume of diluted anesthetic, and the final concentration of local anesthetic. In this case series, the dose of levobupivacaine used was 1.5$2.8 \mathrm{mg} / \mathrm{kg}$, which remains below the maximum allowed dose of $3 \mathrm{mg} / \mathrm{kg}$ (Rioja Garcia, 2015). The minimum volume used was based on Drozdzynska et al., (2017) work in dog's cadavers, which stated that at least $1 \mathrm{ml} /$ $\mathrm{kg}$ of local anesthetic solution is required to cover six intercostal spaces with the deep UGSPB. The minimum concentration of levobupivacaine required to produce sensory blockade is $0.125 \%$ (Casati et al., 2004). Therefore, when local anesthetics are diluted, care must be taken to prevent over-dilution as it may result in ineffective blockade. The differences in volume $(1-1.9 \mathrm{ml} / \mathrm{kg})$ and concentration of local anesthetic used $(0.125 \%-0.25 \%)$ in this case series arise from the retrospective nature of this report. The other serratus plane block studies in people found a direct correlation between the volume of local anesthetic used and the spread of the anesthetic (Bruggink et al., 2012; Biswas et al., 2018b). Although standardization of the volume and concentration is required for future studies in dogs evaluating the effectiveness of UGSPB, it is interesting 
to see the dog that received $0.125 \%$ levobupivacaine did not experience intraoperative nociception. In agreement with Casati et al. (2004), this suggests that a concentration of $0.125 \%$ provides sensory, surgically satisfactory blockade when UGSPB is performed.

The retrospective nature of this case series imposes further limitations: different primary anesthetists were involved in the management of these cases, and the postoperative analgesia protocol was not standardized between patients. Thus, it is not possible to make any conclusions about the impact of this block on the postoperative analgesia requirements. For the purpose of this report we have used universal definition of nociception (20\% increase in cardiovascular parameters in comparison to baseline). However, all the cases reported here had less then 10\% changes in cardiovascular system throughout the surgical procedure. Although this enhances our believe in anti-nociceptive effects of the block performed, cardiovascular changes, despite remaining the gold standard tool for intraoperative nociception assessment, are not without limitations (Ruíz-López et al., 2019). Thus, they need to be interpreted with caution.

The UGSPB may be an alternative loco-regional technique to traditional intercostal nerve blocks (Thompson and Johnson 1991). The benefit of this technique in comparison to standard intercostal nerve blocks is the reduced number of injections and so reduced risk of potential intravascular or intrathoracic punctures. Also, UGSPB as a fascial block presents the possibility of peripheral catheter placement in the interfacial plane for post-operative analgesia management that is impossible with individual intercostal nerve blocks. This technique has been proven effective in humans with multiple rib fractures (Fu et al., 2017). When UGSPB was compared to thoracic paravertebral nerve block in humans, both techniques provided comparable levels of analgesia for 24 hours after thoracotomy (Saad et al., 2018). It might be even equivalent to the thoracic epidural block that remains the gold standard analgesia technique for thoracic procedures in human medicine (Khalil et al., 2017). Thoracic epidural analgesia is still rarely performed in veterinary medicine due to technical difficulty, potential complications (Baidya et al., 2014; Son et al., 2015; Khalil et al., 2017), and lack of clinical studies evaluating its risk-benefit ratio. In light of this, UGSPB as a less technically challenging technique deserves further prospective clinical investigation in dogs undergoing lateral thoracotomies.

\section{Conflict of interest}

The authors declare no conflict of interest.

\section{Author's contribution}

Iago Asorey and Maja Drozdzynska were the anesthetists in charge of the cases. All the authors contributed, largely, to the writing and revision of this manuscript.

\section{References}

Abdallah, F.W., Cil, T., MacLean, D., Madjdpour C., Escallon J., Semple J. and Brull R. 2018. Too deep or not too deep? A propensity-matched comparison of the analgesic effects of a superficial versus deep serratus fascial plane block for ambulatory breast cancer surgery. Reg. Anesth. Pain. Med. 43, 480487.

Baidya D.K., Khanna P. and Maitra S. 2014. Analgesic efficacy and safety of thoracic paravertebral and epidural analgesia for thoracic surgery: a systematic review and meta-analysis. Interact. Cardiovasc. Thorac. Surg. 18, 626-635.

Bailey C.S., Kitchell R.L., Haghighi S.S. and Johnson R.D. 1984. Cutaneous innervation of the thorax and abdomen of the dog. Am. J. Vet. Res. 45, 16891698.

Berg, R.J. and Orton, E.C. 1986. Pulmonary function in dogs after intercostal thoracotomy: comparison of morphine, oxymorphone, and selective intercostal nerve block. Am. J. Vet. Res. 47, 471-474.

Biswas A., Luginbuehl I., Szabo E., Caldeira-Kulbakas M., Crawford M.W. and Everett T. 2018a. Use of serratus plane block for repair of coarctation of aorta: a report of 3 cases. Reg. Anesth. Pain. Med. 43, 641-643.

Biswas A., Castanov V., Li Z., Perlas A., Kruisselbrink R., Agur A. and Chan V. 2018b. Serratus plane block: a cadaveric study to evaluate optimal injectate spread. Reg. Anesth. Pain. Med. 43, 854858.

Blanco R., Parras T., McDonnell J.G. and Prats-Galino A. 2013. Serratus plane block: a novel ultrasoundguided thoracic wall nerve block. Anaesthesia. 68, 1107-1113.

Bruggink S.M, Schroeder K.M., Baker-Herman T.L. and Schroeder C. A. 2012. Weight-based volume of injection influences cranial to caudal spread of local anesthetic solution in ultrasound-guided transversus abdominis plane blocks in canine cadavers. Vet. Surg. 41, 455-457.

Chi-Fei Wang J., Hung C.H., Gerner P., Ji R.R. and Strichartz G.R. 2013. The qualitative hyperalgesia profile: a new metric to assess chronic postthoracotomy pain. Open Pain J. 6, 190-198.

Conzemius M.G., Brockman D.J., King L.G. and Perkowski S.Z. 1994. Analgesia in dogs after intercostal thoracotomy: a clinical trial comparing intravenous buprenorphine and intrapleural bupivacaine. Vet. Surg. 23, 291-298.

Drozdzynska M., Fitzgerald E., Neilson D. and Viscasillas J. 2017. Description of ultrasoundguided serratus plane block in dogs: cadaveric study. Vet. Anaesth. Analg. 44, 389-395.

Durant E., Dixon B., Luftig J., Mantuani D. and Herring A. 2017. Ultrasound- guided serratus plane block for ED rib fracture pain control. Am. J. Emerg. Med. 35, 197 e3-197 e6. 
Evans, H.E. and de Lahunta, A. 2012. Miller's Anatomy of the dog, 4th ed. Saunders, UK, pp: 211-236, 633-636.

Freitag F.A., Gaio T.S., dos Santosa A.A., Muehlbauer E., Machado M., and Duque J.C. 2019. Ultrasoundguided superficial serratus plane block in dog cadavers: an anatomical evaluation and volume dispersion study. Vet. Anesth. Analg. 47, 88-94.

Fu P., Weyker P.D. and Web C.A. 2017. Case report of serratus plane catheter for pain management in a patient with multiple rib fractures and an inferior scapular fracture. A. A. Case Rep., 8. 32-135.

Khalil A.E., Abdallah N.M., Bashandy G.M. and Kaddah T.A. 2017. Ultrasound-guided serratus anterior plane block versus thoracic epidural analgesia for thoracotomy pain. J. Cardiothorac. Vasc. Anesth. 31, 152-158.

Kim D.H., Oh Y.J., Lee J.G., Ha D., Chang Y.J. and Kwak H.J. 2018. Efficacy of ultrasound-guided serratus plane block on postoperative quality of recovery and analgesia after video-assisted thoracic surgery: a randomized, triple-blind, placebocontrolled study. Anesth. Analg. 126, 1353-1361.

Mansour C., Merlin T., Bonnet-Garin J.M. Chaaya R., Mocci R., Conde Ruiz C., Allaouchiche B., Boselli E. and Junot S. 2017. Evaluation of the Parasympathetic Tone Activity (PTA) index to assess the analgesia/nociception balance in anaesthetized dogs. Res. Vet. Sci. 115, 271-277.

Mayes J., Davison E., Panahi P., Patten D., Eljelani F., Womack J. and Varma M. 2016. An anatomical evaluation of the serratus anterior plane block. Anaesth. 71, 1064-1069.

Moores A.L., Halfacree Z.J., Baines S.J. and Lipscomb V.J. 2007. Indications, outcomes and complications following lateral thoracotomy in dogs and cats. J. Small. Anim. Pract. 48, 695-698.

Ökmen, K. and Ökmen, B.M. 2017. The efficacy of serratus anterior plane block in analgesia for thoracotomy: a retrospective study. J. Anesth. 31, 579-585.

Rioja Garcia, E. 2015. Veterinary anesthesia and analgesia, Eds,. Grimm, K.A., Lamont, L.A., Tranquilli, W.J., Greene, S.A. and Roberston, S.A. Oxford, UK: Willey Blackwell, pp: 332-354.
Ruffato, M., Novello, L. and Clark, L. 2015. What is the definition of intraoperative hypotension in dogs? Results from a survey of diplomates of the ACVAA and ECVAA. Vet. Anaesth. Analg. 42, 55-64.

Ruíz-López, P., Domínguez, J. M. and Granados, M. del M. 2019. Intraoperative nociceptionantinociception monitors: a review from the veterinary perspective. Vet. Anaesth. Analg. 47, $152-159$.

Saad F.S., El Baradie S.Y., Aliem M., Ali M.M. and Kotb T.A.M. 2018. Ultrasound-guided serratus anterior plane block versus thoracic paravertebral block for perioperative analgesia in thoracotomy. Saudi J. Anaesth. 12, 565-570.

Son W.G, Jang M., Jo S.M., Yoon J. and Lee I. 2015. The volume effect of lidocaine on thoracic epidural anesthesia in conscious Beagle dogs. Vet. Anaesth. Analg. 42, 414-424.

Stobie D., Caywood D.D., Rozanski E.A., Bing D.R., Dhokarikar P., Raffe M.R., Kannan M.S., King V.L., Hegstad R.L. and Randall D.A. 1995. Evaluation of pulmonary function and analgesia after intercostal thoracotomy and use of morphine administered intramuscularly or intrapleurally and bupivacaine administered intrapleurally. Am. J. Vet. Res. 56, 1098-1109.

Thompson, S.E. and Johnson, J.M. 1991. Analgesia in dogs after intercostal thoracotomy. A comparison of morphine selective intercostal nerve block, and interpleural regional analgesia with bupivacaine. Vet. Surg. 20, 73-77.

Wildgaard K., Ravn J. and Kehlet H. 2009. Chronic postthoracotomy pain: a critical review of pathogenic mechanisms and strategies for prevention. Eur. J. Cardiothorac. Surg. 36, 170-180.

Zocca J.A., Chen G.H., Puttanniah V.G., Hung J.C. and Gulati A. 2017. Ultrasound-guided serratus plane block for treatment of postmastectomy pain syndromes in breast cancer patients: a case series. Pain Pract.17, 141-146. 